\title{
Isolation and Properties of the Mixed
}

\section{Lipid Micelles Present in Intestinal}

\section{Content during Fat Digestion in Man}

\author{
Charles M. Mansbach, II, R. S. Cohen, and P. B. Leff \\ From the Veterans Administration Hospital, and Department of Medicine, \\ Division of Gastroenterology, Duke University Medical Center, \\ Durham, North Carolina 27710
}

A B S T R A C T To evaluate better the physicochemical characteristics of human fat digestion, a method was developed which allowed characterization of the bile acid-lipid mixed micelles of the aqueous phase of postprandial duodenal fluid. Duodenal fluid was collected after a 36-g fat breakfast for two 90-min periods and for $60 \mathrm{~min}$ after i.v. cholecystokinin and was ultracentrifuged at $15,400,000 \mathrm{~g}$-min. The aqueous phase was isolated, passed through a $220-\mathrm{nm}$ filter, and the mixed micelles were concentrated by an ultrafiltration procedure using a $1.5-\mathrm{nm}$ filter. The $1.5-\mathrm{nm}$ retentate was eluted from Sepharose $6 \mathrm{~B}$ columns with $1.5-\mathrm{nm}$ filtrate for both preequilibration fluid and eluent. $1.5-\mathrm{nm}$ filtrate approximated the monomer concentrations. Each sample was assayed for bile acid, fatty acid, lecithin, lysolecithin, protein, cholesterol, and counterions $\left(\mathrm{pH}, \mathrm{Na}^{+}\right.$, $\left.\mathrm{K}^{+}, \mathrm{Ca}^{2+}\right)$. Constituents were concentrated only on the 1.5-nm filter. On gel permeation chromatography, coincident peaks were observed for bile acid, fatty acid, lysolecithin, and cholesterol; and were eluted with a $K_{\text {av }}$ range of $0.50-0.68$ (corresponding to a Stokes radius of $2.3-3.5 \mathrm{~nm}$ ). An average density of 1.25 and coincident peaks of bile acid and fatty acid were found for the mixed micelles on sucrose density gradients. The regression lines of micellar fatty acid, lysolecithin, and cholesterol vs. bile acid gave a stoichiometry of $1.4 \mathrm{~mol}$ fatty acid, $0.15 \mathrm{~mol}$ lysolecithin, and $0.06 \mathrm{~mol}$ cholesterol for each mole of bile acid. Mixed micelles were homogeneous in composition. These results provide direct evidence for the existence of the postprandial nixed micelle and describe several of its physicochemical properties.

Reccived for publication 9 August 1974 and in revised form 28 May 1975.

\section{INTRODUCTION}

The absorption of dietary lipid in the human is an efficient process, over $90 \%$ occurring in the duodenum and proximal jejunum (1). Before its absorption, dietary triglyceride its made more polar via hydrolysis by pancreatic lipase to FFA and partial glycerides. These lipids are partially solubilized in the aqueous intestinal fluid by the detergent action of bile acid (2). The proportion of lipid partitioned into the aqueous phase $(\mathrm{AP})^{1}$ has been shown in humans (3) and rats (4-6) to be correlated with the amount of dietary lipid absorbed. In addition, AP solubilization has been suggested as the rate-limiting step in the absorption of dietary lipid $(4,6)$.

The AP contains isotropically dispersed lipid and is a mixture of bile acid-lipid aggregates in equilibrium with their monomer forms. In previous studies the AP has been isolated from the oil-rich emulsion present in postprandial duodenal fluid by means of ultracentrifugation $(2,7,8)$ and, alternatively, by filtration (9).

Whereas both of these approaches have given useful information about the AP, neither method allows concentration of the mixed micelles. For this reason, it has previously not been possible to study the physicochemical properties of the intestinal bile acid-lipid mixed micelles in detail nor to evaluate the potential role of lysolecithin (10) in the solubilization process.

A method is now described which combines ultracentrifugation, ultrafiltration, and gel permeation chroma-

${ }^{1}$ Abbreviations used in this paper: AP, aqueous phase; $\mathrm{CCK}$, cholecystokinin; CMC, critical micellar concentration; DDF, distal duodenal fluid; $R_{s}$, Stokes radius; TLC, thin-layer chromatography; $V_{0}$, void volume; $V_{t}$, salt volume.

The Journal of Clinical Investigation Volume 56 October 1975.781-791 


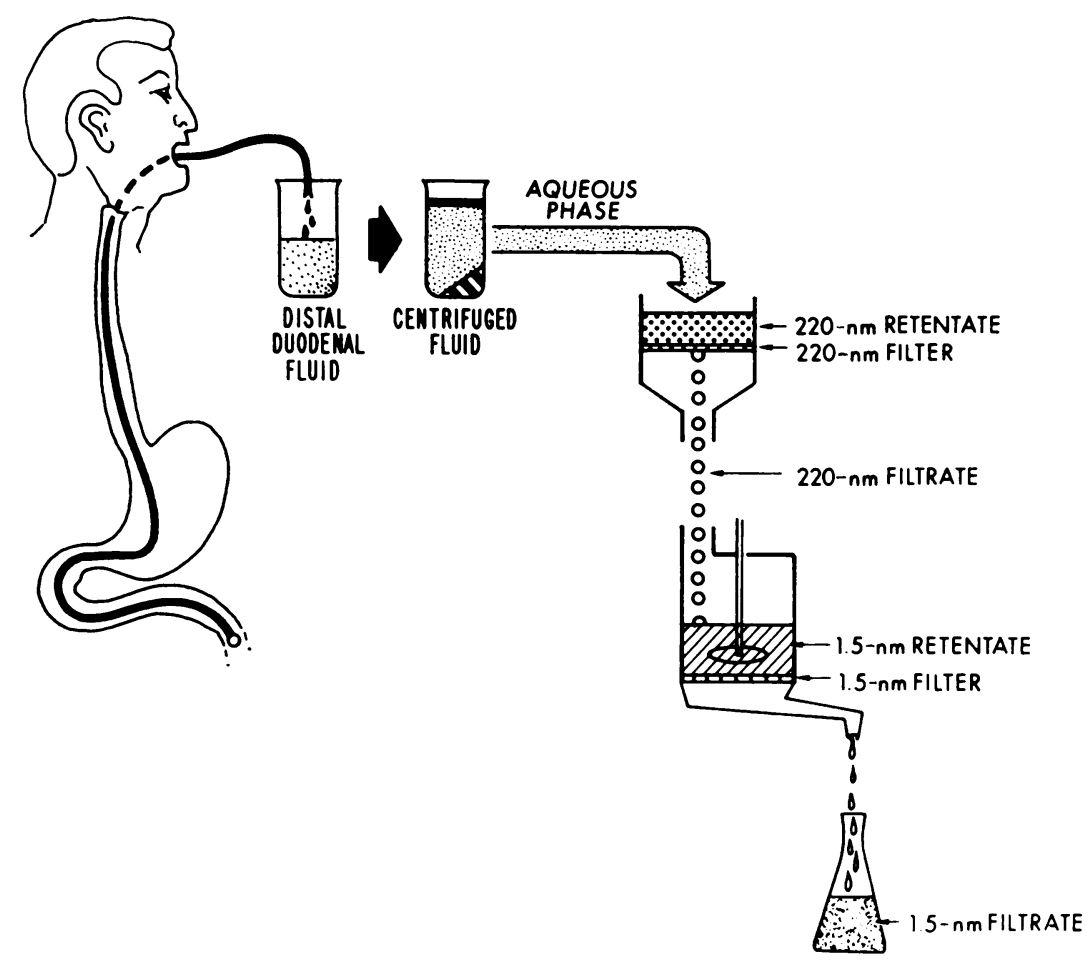

FigUre 1 Schema of separation steps in handling of DDF.

tography of postprandial distal duodenal fluid (DDF) obtained from normal human volunteers. This method allows the development of information concerning the size, density, homogeneity, and stoichiometry of the association between the lipid and bile acid constituents of the postprandial mixed micelle.

\section{METHODS}

Six fasting healthy Caucasian volunteers (four males and two females, age 24-43 yr) were intubated, under fluoroscopic control, to the level of the ligament of Treitz. The tube used was a flexible polyvinyl chloride tube $(4 \mathrm{~mm}$ OD) with a 5-cm length of aspirating ports, just proximal to a terminally placed mercury-filled rubber bag. After the tube was positioned, a breakfast consisting of two fried eggs, two strips of bacon, two slices of toast spread with two pats of butter, and $8 \mathrm{oz}$ of milk was eaten. The meal consisted of $36 \mathrm{~g}$ extractable lipid, the measured constituents of which were: $1.6 \mathrm{~g}$ lecithin, $0.9 \mathrm{~g}$ FFA, $0.7 \mathrm{~g}$ cholesterol, $0.2 \mathrm{~g}$ lysolecithin, and $21.0 \mathrm{~g}$ protein. Postprandial DDF was then collected via siphonage for two $90-$ min periods and then for $45 \mathrm{~min}$ after i.v. infusion of 75 Ivy $U$ of cholecystokinin (CCK) (Karolinska Institute, Stockholm, Sweden). The position of the tube was verified at least once each hour or when the $\mathrm{pH}$ of $\mathrm{DDF}$, which was monitored by $\mathrm{pH}$ paper (pHydrion, Micro Essential Laboratory, Brooklyn, N. Y.) approximately at 15 -min intervals, was $<5$.

All fluid was collected and kept at room temperature during the subsequent separation procedures. The volume of DDF collected ranged between 90 and $110 \mathrm{ml}$ per collection period. Samples of DDF from each period were centrifuged at $60,000 \mathrm{rpm}$ for $1 \mathrm{~h}$ in a Spinco model $60 \mathrm{Ti}$ rotor (Beckman Instruments, Inc., Spinco Div., Palo Alto, Calif.) $(15,400,000 \mathrm{~g}$-min) with a Beckman ultracentrifuge (model L2 65B). To evaluate the presence of a significant bile acid gradient developing in the AP as a result of centrifugation, two samples of DDF were centrifuged as described and five serial samples of AP from the top to the bottom of the centrifuge tube were obtained. The bile acid concentrations varied between 10 and $14 \%$ greater in the bottom-most as compared to the upper-most samples. After centrifugation, the centrifuge tube contained a small pellet at the bottom, a large clear AP, and a small oil phase at the top, which consisted of both liquid and solid components. The liquid, but not the solid component, was completely soluble in chloroform: methanol $(2: 1, \mathrm{vol} / \mathrm{vol})$.

The entire AP was aspirated and passed through a 220-nm filter (Millipore Corp., Bedford, Mass.) to enable its subsequent ultrafiltration. The 220-nm filtrate was passed through a $1.5-\mathrm{nm}$ filter (UM 10, Amicon Corp., Scientific Sys. Div., Lexington, Mass.) with a model 52 Amicon filter holder in a $\mathrm{N}_{2}$ atmosphere at $40 \mathrm{lb} / \mathrm{in}^{2}$. Because pure bile acid micelles have a Stokes radius of approximately $2 \mathrm{~nm}(11,12)$ it was hypothesized that bile acid-lipid mixed micelles, if present, would pass through the $220-\mathrm{nm}$ filter and be concentrated by the $1.5-\mathrm{nm}$ filter and that the micelles' constituents would be found in their monomer form in the $1.5-\mathrm{nm}$ filtrate. A schema of these separation steps is shown in Fig. 1. Gel permeation chromatography was then performed with Sepharose 6B (Pharmacia Fine Chemicals, Inc., Piscataway, N. J.) columns $(6 \times 53 \mathrm{~mm})$. Each column was preequilibrated with $15 \mathrm{ml}$ of $1.5-\mathrm{nm}$ filtrate. $0.5 \mathrm{ml}$ of $1.5-\mathrm{nm}$ retentate was then applied to the top of the column. Additional $1.5-\mathrm{nm}$ filtrate was used as eluent. A Technicon (AA1) peristaltic pump (Technicon Instru- 
ments Corp., Tarrytown, N. Y.) was used to maintain a constant flow rate of $1 \mathrm{ml} / 4.5 \mathrm{~min}$. Fractions timed to yield $1 \mathrm{ml}$ were collected. This method circumvented the variation in drop size which occurs with detergent solutions of varying concentration. The void volume $\left(V_{0}\right)$ as determined by elution of Blue Dextran 2000 and salt volume $\left(V_{t}\right)$ as determined by elution of dextrose were ascertained in each instance by dissolving a small amount of each in the $1.5-\mathrm{nm}$ retentate applied to the column. Collections were carried out for $3 \mathrm{ml}$ beyond $V_{t}$. Recovery of applied material was 91 $\pm 2.6 \%$, implying no significant binding to the Sepharose.

Sucrose density centrifugation of the micelles was performed with a $30-18 \%$ sucrose linear density gradient. On top of the gradient was first layered $0.2 \mathrm{ml}$ of $1.5-\mathrm{nm}$ retentate, followed by $5 \%$ sucrose. $0.2 \mathrm{ml}$ of $60 \%$ sucrose was used as a cushion. All sucrose solutions were made up by dissolving the sucrose in $1.5-\mathrm{nm}$ filtrate. This gradient was spun in a SW50L rotor (Beckman Instruments, Inc.) at $45,000 \mathrm{rpm}$ for $12 \mathrm{~h}$. The bottom of the tube was pierced by a needle and 22 fractions were collected. Sucrose density was determined by using a Bausch \& Lomb refractometer (Bausch \& Lomb Inc., Scientific Optical Products Div., Rochester, N. Y.). Fractions were then assayed for total bile acid and FFA.

DDF and AP from all three collection periods, as well as all filtration derivatives and column fractions, were frozen approximately $15 \mathrm{~h}$ after the start of the DDF collection. These specimens were subsequently analyzed for bile acid, FFA, lecithin, lysolecithin, cholesterol, protein, and counterions $\left(\mathrm{N}^{+}, \mathrm{K}^{+}, \mathrm{Ca}^{2+}\right.$, and $\left.\mathrm{pH}\right)$. To evaluate the effect of prolonged incubation at room temperature, paired 1-ml samples of both DDF and AP were obtained from each of the three periods. One member of each pair was immediately frozen and the other was allowed to stand at room temperature, along with all routine samples. Subsequent assays of these paired aliquots for lecithin, lysolecithin, monoglyceride, and FFA were performed.

Bile acid concentrations were determined by the steroid dehydrogenase method essentially as described by Stempfel and Sidbury (13). The accuracy of this assay was improved by reading the optical density at $340 \mathrm{~nm}$ both before and after addition of the enzyme. This assay, as well as all other assays performed, was carried out within a previously determined linear range.

Lipids were extracted by the method of Folch et al. (14) ; $1 \mathrm{~N} \mathrm{HCl}$ was used to break the phases. The chloroform-rich phase was reduced to dryness and was then brought up in chloroform: methanol $(2: 1, \mathrm{vol} / \mathrm{vol})$ and stored at $-20^{\circ} \mathrm{C}$ until analysis.

FFA was assayed phototitrametrically in a phenol redsodium barbital-heptane buffer solution at $560 \mathrm{~nm}$ as suggested by Mozinger (15)

Monoglycerides were isolated by thin-layer chromatography (TLC) on silica gel G layers $500 \mu \mathrm{m}$ thick by using ethyl ether: petroleum ether: acetic acid $(50: 50: 1$ by vol) and quantitated by the method of Amenta (16).

Lecithin and lysolecithin were separated by means of TLC. This was performed on silica gel G layers $500 \mu \mathrm{m}$ thick, by using chloroform: methanol: ammonium hydroxide $: \mathrm{H}_{2} \mathrm{O}$ (150:75:9:3 by vol). The phospholipids were eluted from the gel with chloroform: methanol $(1: 1)$, and the eluent evaporated under a stream of $\mathrm{N}_{2}$. The phospholipids were digested with $10 \% \mathrm{Mg}\left(\mathrm{NO}_{3}\right)_{2}$, dried, ashed, and acid hydrolyzed to convert any pyrophosphate to inorganic phosphate, as suggested by Chen et al. (17). The phosphate content was then measured by the method of Ames and
Dubin (18). Lecithin and lysolecithin from egg yolk (19) were used as standards.

Total cholesterol was assayed by the method of Abell et al. (20), and total protein was assayed by the method of Lowry et al. (21).

Sodium, potassium, and chloride were measured by flame photometry; calcium was measured by the method of Kessler and Wolfman (22). $\mathrm{pH}$ was measured on a Corning model $12 \mathrm{pH}$ meter (Corning Scientific Instruments, Medfield, Mass.).

The Sepharose 6B columns were calibrated by chromatographing five proteins (aldolase, bovine serum albumin, chymotrypsinogen A, cytochrome $c$, and ovalbumin), at a concentration of $2 \mathrm{mg} / \mathrm{ml}$, in $0.1 \mathrm{M}$ phosphate buffer at $\mathrm{pH}$ 7.2. 0.1 $\mathrm{M}$ phosphate buffer was used for both preequilibration and elution. $0.25-\mathrm{ml}$ fractions were collected and assayed for protein at $280 \mathrm{~nm}$ on a Gilford spectrophotometer (Gilford Instrument Laboratories, Inc., Oberlin, Ohio). Observed $K_{\mathrm{av}}$ 's were converted to their negative error function complements as suggested by Ackers (23). These in turn, were plotted against the proteins' Stokes radii $\left(R_{B}\right)$, (24). The resultant curve: $y=44.4 x+5.47, \mathrm{SE}=2.3$, $r=0.99$, was then used to calculate the $R_{s}$ of the bile acidlipid mixed micelles. It has recently been shown that the slope of the regression line of the curve describing the relationship between the $K_{\mathrm{av}}$ and $R_{\mathrm{s}}$ for proteins chromatographed on gel columns is the same, whether eluted with inorganic buffer or detergent solutions (24). Thus the above curve was used to infer $R_{s}$ from the experimentally derived $K_{\mathrm{av}}$ 's of the bile acid-lipid mixed micelles.

Statistical methods. One-way analysis of variance with Sheffé contrasts (25), a type of multivariate analysis in which each group of variables is compared to every other variable, and determination of regression lines by the leastsquares method were performed on an IBM model 1130 computer (International Business Machines Corp., Armonk, N. Y.).

\section{RESULTS}

Concentrations of the constituents assayed appear in Table I, showing the relative concentrations in DDF and in the various separation stages before gel column chromatography. These values are displayed for all three collection periods. As can be seen from the data, concentrations of lecithin were insignificant in comparison to all other constituents, and lecithin data, per se, will not be commented upon further.

There was a decrement in concentration of all AP constituents compared to their DDF values. Whereas no concentration of any constituent was observed at the 220-nm filter, each constituent was greatly retarded by the $1.5 \mathrm{~nm}$ membrane during all three collection periods, suggesting the presence of aggregates whose diameters are at least $1.5 \mathrm{~nm}$. As the aggregated species are in continuous rapid equilibrium with their respective monomers, each of whose concentration approximates the critical micellar concentration (CMC) for that species, the ultrafiltrate was assumed to contain the constituents at concentrations close to their CMC. Recovery across the $1.5-\mathrm{nm}$ filter for all constituents averaged $96 \pm 2 \%$, suggesting no significant adsorption of constituents to the filter. 
TABLE I

Lipid and Protein Concentrations in DDF, AP, and Filtration Stages in Six Normals*

\begin{tabular}{|c|c|c|c|c|c|c|c|c|}
\hline $\begin{array}{l}\text { Time after meal } \\
\text { (Average } \mathrm{pH} \text { ) }\end{array}$ & Stage & Total bile acid & FFA & Monoglyceride & Lysolecithin & Lecithin & Cholesterol & Protein \\
\hline & & \multicolumn{6}{|c|}{$\mu \mathrm{mol} / \mathrm{ml}$} & $m g / m l$ \\
\hline \multirow{6}{*}{$\begin{array}{l}0-90 \min \\
\quad(\mathrm{pH} \mathrm{5.7)}\end{array}$} & DDF & $9.3 \pm 0.75$ & $28.3 \pm 4.1$ & $2.53 \pm 1.2$ & $2.4 \pm 0.35$ & $0.07 \pm 0.04$ & $1.5 \pm 0.12$ & $16.1 \pm 1.2$ \\
\hline & AP & $6.4 \pm 0.78$ & $9.9 \pm 1.9$ & $0.93 \pm 0.50$ & $0.88 \pm 0.18$ & $0.06 \pm 0.05$ & $0.30 \pm 0.06$ & $9.6 \pm 1.3$ \\
\hline & $22 \mathrm{R} \ddagger$ & $6.5 \pm 0.79$ & $10.5 \pm 2.0$ & & $0.88 \pm 0.18$ & $0.05 \pm 0.02$ & $0.41 \pm 0.06$ & $10.2 \pm 1.1$ \\
\hline & $22 \mathrm{~F}$ & $6.4 \pm 0.71$ & $8.9 \pm 1.5$ & & $0.79 \pm 0.14$ & $0.01 \pm 0.01$ & $0.28 \pm 0.06$ & $8.9 \pm 1.2$ \\
\hline & $1.5 \mathrm{R}$ & $49.2 \pm 9.4$ & $54.8 \pm 8.8$ & $4.62 \pm 2.7$ & $6.4 \pm 0.98$ & $0.19 \pm 0.10$ & $2.4 \pm 0.06$ & $56.2 \pm 11.2$ \\
\hline & $1.5 \mathrm{~F}$ & $1.7 \pm 0.20$ & $2.3 \pm 0.47$ & $0.12 \pm 0.05$ & $0.18 \pm 0.07$ & $0.01 \pm 0.01$ & $0.01 \pm 0.01$ & $5.0 \pm 0.94$ \\
\hline \multirow{6}{*}{$\begin{array}{r}90-180 \text { min } \\
\quad(\mathrm{pH} 5.2)\end{array}$} & DDF & $5.0 \pm 0.87$ & $20.7 \pm 2.7$ & & $1.7 \pm 0.28$ & $0.16 \pm 0.07$ & $1.1 \pm 0.04$ & $14.4 \pm 1.2$ \\
\hline & AP & $2.7 \pm 2.0$ & $4.0 \pm 1.3$ & & $0.22 \pm 0.13$ & $0.01 \pm 0.01$ & $0.08 \pm 0.01$ & $9.7 \pm 0.82$ \\
\hline & $22 \mathrm{R}$ & $2.7 \pm 2.2$ & $4.3 \pm 0.16$ & & $0.19 \pm 0.13$ & $0.01 \pm 0.01$ & $0.08 \pm 0.06$ & $10.2 \pm 0.86$ \\
\hline & $22 \mathrm{~F}$ & $2.5 \pm 0.80$ & $3.1 \pm 1.1$ & & $0.17 \pm 0.13$ & $0.01 \pm 0.01$ & $0.05 \pm 0.05$ & $9.6 \pm 0.79$ \\
\hline & $1.5 \mathrm{R}$ & $14.1 \pm 8.8$ & $17.6 \pm 8.9$ & & $1.5 \pm 1.3$ & $0.03 \pm 0.02$ & $0.48 \pm 0.41$ & $50.6 \pm 2.8$ \\
\hline & $1.5 \mathrm{~F}$ & $1.1 \pm 0.20$ & $1.5 \pm 0.27$ & & $0.05 \pm 0.03$ & $0.03 \pm 0.02$ & $0.01 \pm 0.01$ & $4.4 \pm 1.0$ \\
\hline \multirow{6}{*}{$\begin{array}{c}180-240 \mathrm{~min} \\
\quad(\text { post-CCK) } \\
(\mathrm{pH} \mathrm{6.0)}\end{array}$} & DDF & $8.0 \pm 1.2$ & $21.5 \pm 4.7$ & $1.04 \pm 0.07$ & $1.4 \pm 0.23$ & $0.05 \pm 0.02$ & $0.80 \pm 0.07$ & $8.9 \pm 1.0$ \\
\hline & AP & $7.1 \pm 1.5$ & $9.4 \pm 2.2$ & $0.31 \pm 0.03$ & $1.0 \pm 0.28$ & $0.02 \pm 0.02$ & $0.33 \pm 0.08$ & $5.8 \pm 0.63$ \\
\hline & $22 \mathrm{R}$ & $7.0 \pm 1.4$ & $10.8 \pm 2.5$ & & $1.0 \pm 0.33$ & $0.03 \pm 0.01$ & $0.32 \pm 0.12$ & $6.4 \pm 0.69$ \\
\hline & $22 \mathrm{~F}$ & $7.1 \pm 1.7$ & $10.0 \pm 2.4$ & & $1.0 \pm 0.28$ & $0.02 \pm 0.01$ & $0.31 \pm 0.08$ & $5.9 \pm 0.72$ \\
\hline & $1.5 \mathrm{R}$ & $78.9 \pm 23.7$ & $61.0 \pm 15.3$ & $4.02 \pm 2.2$ & $9.5 \pm 3.6$ & $0.07 \pm 0.03$ & $3.2 \pm 0.91$ & $35.8 \pm 3.0$ \\
\hline & $1.5 \mathrm{~F}$ & $1.4 \pm 0.37$ & $2.0 \pm 0.22$ & $0.003 \pm 0.003$ & $0.18 \pm 0.06$ & $0.02 \pm 0.01$ & $0.02 \pm 0.01$ & $2.7 \pm 0.21$ \\
\hline
\end{tabular}

* The data shown represent the mean $\pm \mathrm{SE}$.

$\ddagger 22 \mathrm{R}, 220$-nm retentate; $22 \mathrm{~F}, 220$-nm filtrate; $15 \mathrm{R}, 1.5$-nm retentate; $15 \mathrm{~F}, 1.5$-nm filtrate as described in Methods.

Since large concentration gradients were developed at the $1.5-\mathrm{nm}$ membrane, the $1.5-\mathrm{nm}$ filtrate concentrations have the potential of being greater than their true CMC value (26). However, when tested in an in vitro setting, the ultrafiltrate concentration of taurodeoxycholate was found to be $0.68 \mathrm{mM}$ and taurocholate $2.9 \mathrm{mM}$ in $0.15 \mathrm{M} \mathrm{NaCl}$ (theory, 0.8 and $3.2 \mathrm{mM}$, respectively) (27). The filtrate from these in vitro experiments was tested serially for bile acid concentration during filtration. Only the last drops filtered were found to have an increased bile acid concentration (as compared to the first drops), which was $155 \%$ of the initial values.

Table II shows the amount of lipolysis and phospholipolysis occurring while specimens remain at room

TABLE II

Lipolysis and Phospholipolysis Occurring during Manipulations of Duodenal Fluid at Room Temperature*

\begin{tabular}{lccc}
\hline & \multicolumn{2}{c}{$\begin{array}{c}\text { Lysolecithin as percent of the total } \\
\text { of lecithin and lysolecithin }\end{array}$} \\
\cline { 2 - 4 } $\begin{array}{c}\text { Percent increment of } \\
\text { FFA, } 22^{\circ} \mathrm{C} \text { vs. } \\
\text { immediately frozen }\end{array}$ & $\begin{array}{c}\text { Immediately } \\
\text { frozen }\end{array}$ & Left at $22^{\circ} \mathrm{C}$ \\
\hline $\mathrm{DDF}$ & $35.4 \% \pm 7.4 \mathrm{SE}$ & $54.5 \% \pm 5.6$ & $90.5 \% \pm 2.5$ \\
$\mathrm{AP}$ & $20.4 \% \pm 6.5 \mathrm{SE}$ & $81.3 \% \pm 10.0$ & $90.5 \% \pm 7.4$ \\
\hline
\end{tabular}

* The data represent the mean percentage $\pm \mathrm{SE}$ of six normals. temperature during the course of the experiment. Paired aliquots of immediately frozen and unfrozen specimens are compared (see Methods).

The degree of lipolysis is represented by the FFA data, which showed in DDF an increment with time of $35 \%$ and, in the AP, an increment of $20 \%$. This suggests that, whereas lipolysis in intraluminal fluid outside the patient does occur, it accounts for a minority of the total fatty acid present. Although observations of monoglyceride were more limited (four pairs, DDF only), an average reduction of $39 \%$ was found in the quick-frozen as compared to the unfrozen samples. Phospholipolysis was evaluated by comparing relative concentrations of lecithin and lysolecithin in paired aliquots (frozen vs. unfrozen). This data is summarized in columns 2 and 3 of Table II, which shows in $\mathrm{DDF}$ and to a lesser extent in $\mathrm{AP}$ an increment of lysolecithin in the unfrozen compared to the frozen sample.

Counterions, in the form of sodium, potassium, and calcium, were measured in DDF, AP, $1.5-\mathrm{nm}$ retentate, and $1.5-\mathrm{nm}$ filtrate; the values appear in Table III. As anticipated from the increment of anions, all counterions measured were increased in the $1.5-\mathrm{nm}$ retentate. The most prominent counterion in the DDF, and in all separation stages measured, was sodium. However, calcium was partitioned into the $1.5-\mathrm{nm}$ retentate out 
of proportion to $\mathrm{Na}^{+}$and $\mathrm{K}^{+}$and, as a consequence, was relatively less prominent in the $1.5-\mathrm{nm}$ filtrate than the other two cations. This effect was most marked in the 0-90-min collection period, where DDF- $\mathrm{Ca}^{2+}$ concentration was highest, having come from the recently ingested $8 \mathrm{oz}$ of milk. For all three collection periods the concentration gradient for $\mathrm{Ca}^{2+}$ across the $1.5-\mathrm{nm}$ membrane was 3-5-fold, whereas that for $\mathrm{Na}^{+}$and $\mathrm{K}^{+}$ was 1.1-1.6-fold. Data obtained when taurodeoxycholate and linoleate were filtered together with $\mathrm{NaCl}$ and $\mathrm{Ca}^{2+}$ in an in vitro experiment is shown in the legend to Table III. The concentration gradient for $\mathrm{Na}^{+}$across the $1.5-\mathrm{nm}$ membrane was 1.1 and for $\mathrm{Ca}^{2+}$ was 2.5

Fluid at all stages of separation and filtration was observed through a polarizing light microscope with $100 \times, 200 \times$, and $400 \times$ magnification. Small birefringent "Maltese crosses," suggestive of smectic aggregations of lipid, were seen in substantial concentrations in all DDF samples when viewed with crossed polarizers. These birefringent aggregates were absent or rare in AP specimens and were sometimes seen in the $220-\mathrm{nm}$ retentate, but were seen neither in the $220-\mathrm{nm}$ filtrate, the $1.5-\mathrm{nm}$ retentate, nor the $1.5-\mathrm{nm}$ filtrate specimens. The composition of these smectic lipids is unknown since their concentration was so low that the lipid parameters measured were not influenced by their presence (compare $220-\mathrm{nm}$ retentate to $220-\mathrm{nm}$ filtrate, Table I).

TABLE III

Counterion Concentrations in DDF and Derivitizes*

\begin{tabular}{llcrr}
\hline \multicolumn{2}{c}{ Time after meal } & Sodium & Potassium & \multicolumn{1}{c}{ Calcium } \\
\hline \multirow{3}{*}{$0-90 \mathrm{~min}$} & & & \multicolumn{1}{l}{ meq/liter } \\
& DDF & $105 \pm 4.2$ & $14.4 \pm 1.0$ & $14.9 \pm 2.6$ \\
& AP & $110 \pm 3.1$ & $14.8 \pm 1.1$ & $10.3 \pm 2.7$ \\
& $1.5 \mathrm{R} \ddagger$ & $145 \pm 9.0$ & $21.3 \pm 2.0$ & $30.9 \pm 6.1$ \\
& $1.5 \mathrm{~F}$ & $97 \pm 7.5$ & $13.2 \pm 1.5$ & $7.1 \pm 2.1$ \\
$90-180 \mathrm{~min}$ & $\mathrm{DDF}$ & $103 \pm 1.0$ & $12.8 \pm 0.7$ & $8.0 \pm 0.7$ \\
& $\mathrm{AP}$ & $110 \pm 3.7$ & $13.5 \pm 0.6$ & $7.3 \pm 1.2$ \\
& $1.5 \mathrm{R}$ & $121 \pm 11.5$ & $15.1 \pm 0.6$ & $17.2 \pm 2.0$ \\
& $1.5 \mathrm{~F}$ & $106 \pm 3.0$ & $13.0 \pm 1.0$ & $5.7 \pm 1.2$ \\
$180-240 \mathrm{~min}$ & $\mathrm{DDF}$ & $114 \pm 3.7$ & $9.5 \pm 0.6$ & $4.6 \pm 1.1$ \\
(post-CCK) & $\mathrm{AP}$ & $122 \pm 5.6$ & $10.1 \pm 0.6$ & $4.3 \pm 1.0$ \\
& $1.5 \mathrm{R}$ & $182 \pm 17.9$ & $15.5 \pm 0.5$ & $15.4 \pm 2.4$ \\
& $1.5 \mathrm{~F}$ & $113 \pm 5.7$ & $9.5 \pm 0.6$ & $3.2 \pm 1.1$ \\
\hline
\end{tabular}

In vitro data: When $9 \mathrm{mM}$ taurodeoxycholate and $9 \mathrm{mM}$ linoleate were filtered through a UM 10 membrane with 139 meq/liter $\mathrm{NaCl}$ and $5 \mathrm{meq} /$ liter $\mathrm{CaCl}^{2+}$ in $0.036 \mathrm{M}$ Tris buffer, $\mathrm{pH} 6.5$, the ultrafiltrate contained $136 \mathrm{meq} /$ liter $\mathrm{Na}^{+}$and 4.2 meq/liter $\mathrm{Ca}^{2+}$, and the retentate contained $149 \mathrm{meq} /$ liter $\mathrm{Na}^{+}$and $11.5 \mathrm{meq} /$ liter $\mathrm{Ca}^{2+}$.

* The data represent the mean $\pm \mathrm{SE}$ of six normal subjects. $\ddagger$ Abbreviations are the same as those used in Table $I$.

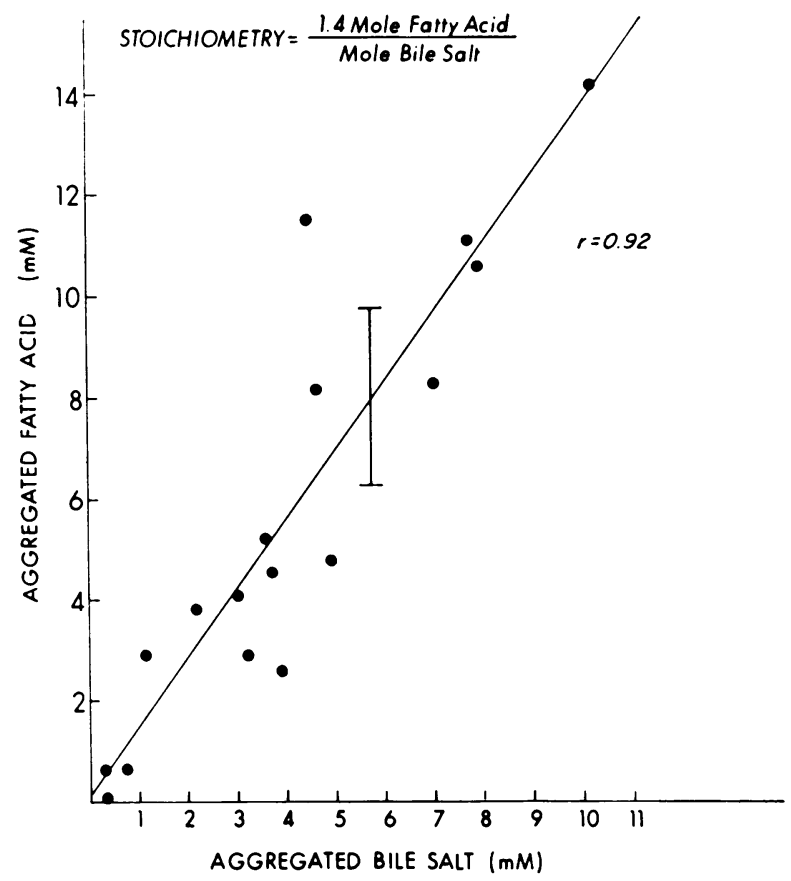

FIGURE 2 Relationship between aggregated fatty acid and aggregated bile acid. The concentration in the aggregated form was determined by substracting the $1.5-\mathrm{nm}$ filtrate concentration from the corresponding $220-\mathrm{nm}$ filtrate concentration. The slope of the regression line, whose correlation coefficient is indicated by $r$, is taken to be the stoichiometry of the association between the variables. The vertical bar indicates the SE of the estimate.

The $220-n m$ filtrate contains a mixture of both the bile acid-lipid mixed micelles and the appropriate monomer phase of each constituent, whereas the $1.5-\mathrm{nm}$ filtrate contains only the latter. Therefore, the subtraction of a species $1.5-\mathrm{nm}$ filtrate concentration from its 220 -nm filtrate concentration yields the aggregated, or micellar concentration. By plotting the micellar concentrations of the lipid constituents against the micellar concentration of bile acid, for all six subjects and all three collection periods, curves were constructed whose slopes described the stoichiometry of the lipid-bile acid associations. The relationship between protein and bile acid was viewed in a similar manner.

Figs. 2-4 show the relationship of micellar FFA, lysolecithin, and cholesterol, respectively, to micellar bile acid plotted as just described. It can be seen that there is a significant and positive correlation of each lipid with bile acid. The slopes of the regression lines describing these relationships show that for each mole of bile acid there are $1.4 \mathrm{~mol}$ of FFA, $0.15 \mathrm{~mol}$ of lysolecithin, and $0.06 \mathrm{~mol}$ of cholesterol. Furthermore, the confidence limits of the regression lines include the origin, suggesting that in the absence of bile acid aggregates there are few lipid aggregates present. 


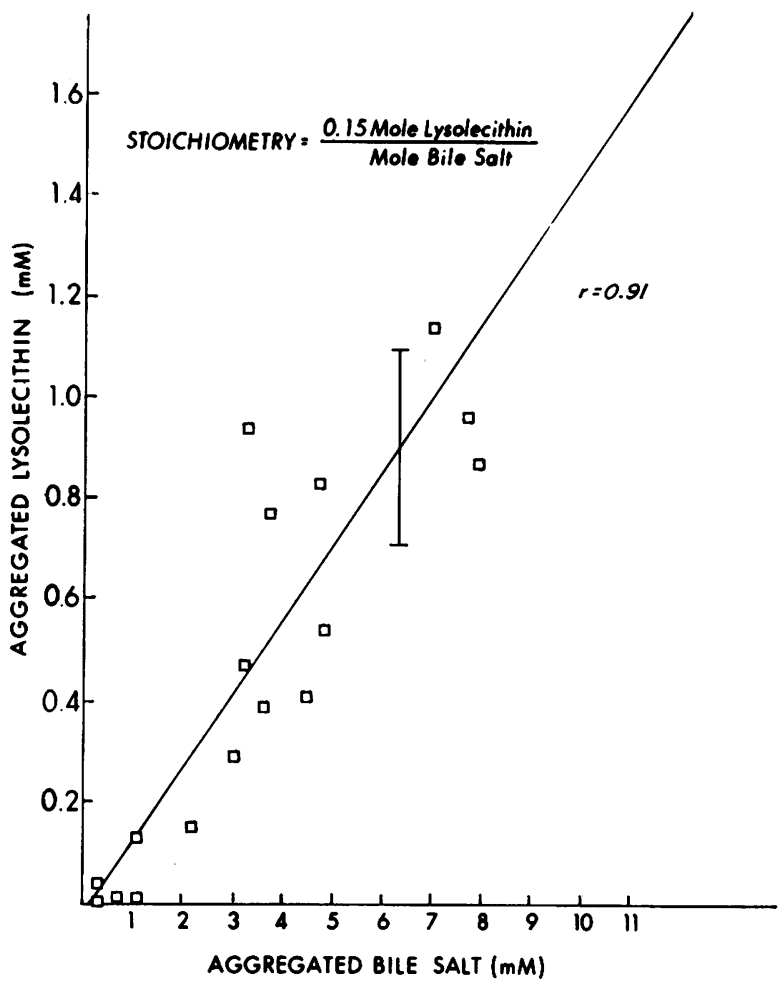

FIGURE 3 Relationship between aggregated bile acid and aggregated lysolecithin. The concentration of both variables in aggregated form was determined as in Fig. 2. The slope of the regression line, whose correlation coefficient is $r$, is indicated by the stoichiometry of the association. The vertical bar indicates the $\mathrm{SE}$ of the estimate.

The relationship between micellar bile acid and protein is shown in Fig. 5. It is evident that there is no correlation between these two variables.

The results of gel permeation chromatography of the bile acid-lipid mixed micelles of a representative subject (at $0-90 \mathrm{~min}$ ) are depicted in Fig. 6. The values before the void volume $V_{0}$ are greater than zero because the column was preequilibrated with $1.5-\mathrm{nm}$ filtrate. The coincidence of concentration peaks of FFA, bile acid, and lysolecithin (and cholesterol when analyzed) shown in Fig. 6 was typical for all subjects. The coincidence of these peaks suggests that these constituents were associated in similar-sized particles. By contrast, protein reached a peak value at the salt volume $\left(V_{t}\right)$, suggesting that its particle size was considerably smaller than that of the bile acid-lipid mixed micelles, and that the bulk of the protein was not associated with the bile acid-lipid aggregates.

The degree of penetration of the gel by the bile acidlipid mixed micelles depicted in Fig. 6 is reflected by a $K_{\mathrm{av}}$ of 0.56 . When compared to the $K_{\mathrm{av}}$ of the calibration proteins (see Methods) this $K_{\text {ar }}$ suggests a Stokes radius of $3.1 \mathrm{~nm}$. Similar comparisons of con- centration peaks from the chromatograms of all six subjects show a range of aggregate size of from 2.3-3.5 $\mathrm{nm}$ corresponding to a molecular weight of 27,000 69,000 .

Collection periods whose $\mathrm{pH}$ was less than 5.2 had little or no bile acid or lipid in aggregated form and these chromatograms had no concentration peaks. This observation may be explained by the fact that, at these $\mathrm{pH}$ 's, bile acids, especially those conjugated with glycine and thus with a pKa of 4 (28), become less ionized. In the protonated state they are themselves unable to form micelles and therefore function less efficiently as detergents. An alternate explanation for the lack of concentration peaks is that a reduced bile acid concentration was present at these $\mathrm{pH}$ 's. This possibility is rendered less likely by the finding that one sample whose total bile acid concentration was $3.6 \mathrm{mM}$ did have a definable concentration peak on exclusion chromatography.

If the stoichiometric data present in Figs. 2-4 accurately reflect the relationship of lipid constituents to bile acid in the mixed micelle, similar ratios should be obtainable when the relationship between the lipid constituents and bile acid are calculated from the chromatograms. By using the concentration peaks (1.5$\mathrm{nm}$ filtrate concentration subtracted) as a representative sample of the micelles, the following ratios of lipid to bile acid were obtained : $1.5 / 1 \pm 0.14, \mathrm{FFA} ; 0.12 / 1 \pm$

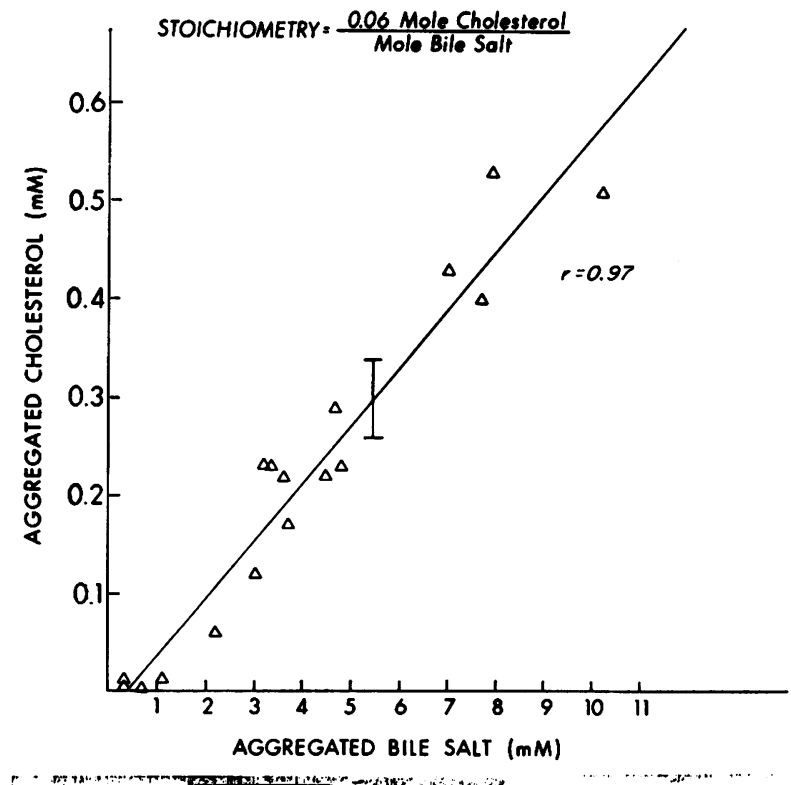

FIGURE 4 Relationship between aggregated bile acid and aggregated cholesterol. The concentration of both variables in an aggregated form was determined as in Fig. 2. The slope of the regression line, whose correlation coefficient is $r$, is indicated by the stoichiometry of the association. The vertical bar indicates the $\mathrm{SE}$ of the estimate. 


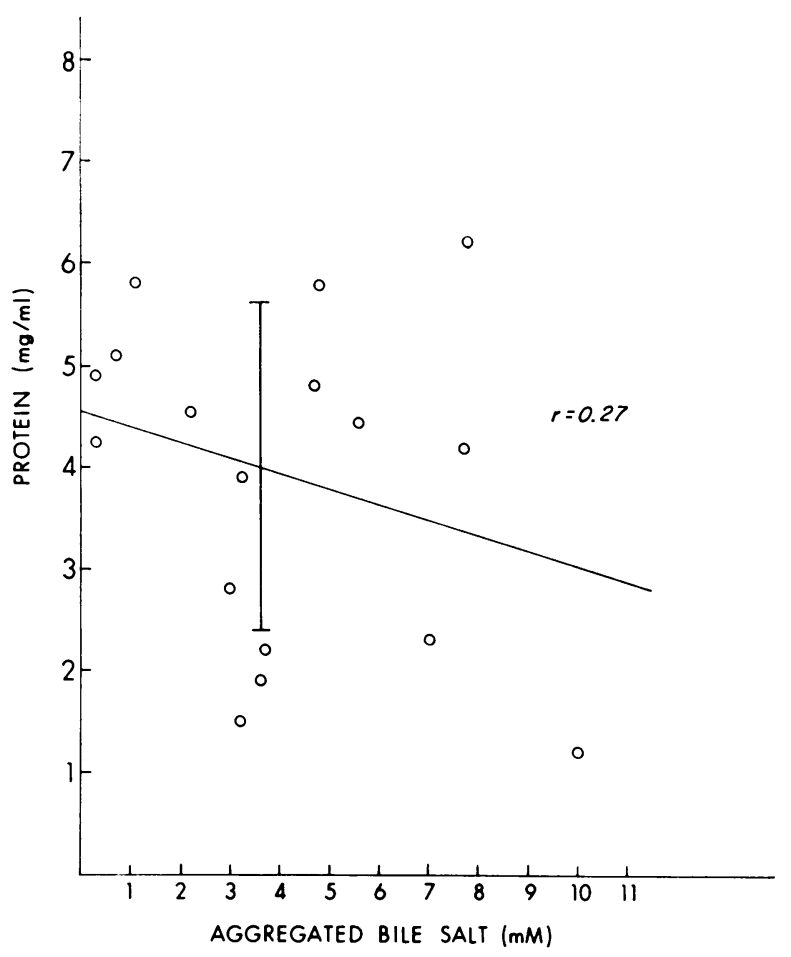

FIGURE 5 Relationship between aggregated bile acid and aggregated protein. The concentration of both variables in aggregated form was determined as in Fig. 2. There is no significant correlation $(r)$ between the variables, therefore no stoichiometry of association is indicated. The vertical bar indicates the $\mathrm{SE}$ of the estimate.

0.02 , lysolecithin; and $0.03 / 1 \pm 0.003$, cholesterol. These values approach, and thus confirm the accuracy of, the data derived from the stoichiometric relationships presented in Figs. 2-4. In addition, these concordant rela-

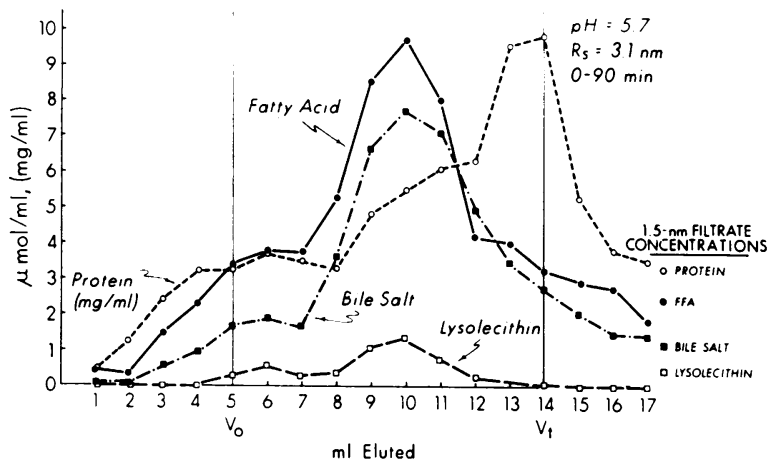

Figure 6 A representative chromatogram of $1.5-\mathrm{nm}$ retentate on Sepharose 6B. $1.5-\mathrm{nm}$ filtrate was used to preequilibrate the column and was also used as the eluent. The elution volume is indicated on the abscissa. The vertical bars indicate the void $\left(V_{0}\right)$ and salt volumes $\left(V_{t}\right)$ of the column. The symbols at the right of the figure indicate the $1.5-\mathrm{nm}$ filtrate concentration of each constituent analyzed. The $\mathrm{pH}$ of the whole DDF and Stokes radius, $R_{s}$, is shown.

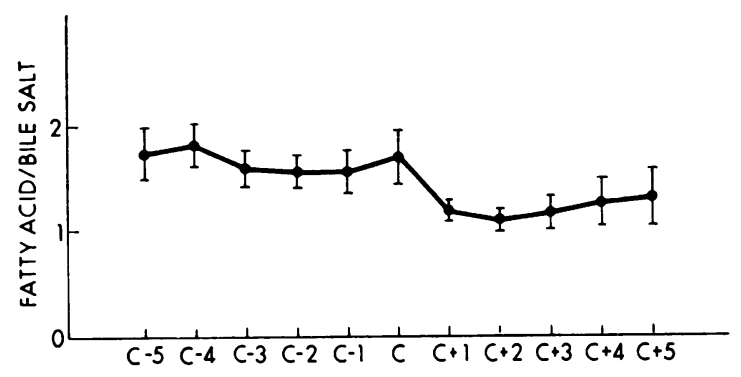

Figure 7 Fatty acid/bile acid ratios of 1.5 -nm retentate chromatographed on Sepharose 6B. The mean and SE, indicated by the vertical bars, are shown for each chromatogram in which there was a definable bile acid concentration peak. The concentration peak is indicated by "C." "C- 5 " through " $\mathrm{C}-1$ " indicate the milliliters of elution from the void volume to the concentration peak. " $\mathrm{C}+1$ " through " $\mathrm{C}+5$ " indicate the milliliters of elution from the concentration peak to or beyond the salt volume of the column. The multivariate $P$ value was 0.0782 . The Scheffé contrasts showed no significant difference between any two variables. The lowest univariate $P$ value, 0.71 , was found on contrasting $\mathrm{C}-4$ with $\mathrm{C}+2$.

tionships suggest that the micelle maintains its integrity throughout the concentration and chromatography steps.

Data relating to the homogeneity of composition of the mixed micelles appear in Fig. 7 and are expressed as the molar ratio of FFA/bile acid. A composite of the fatty acid/bile acid ratios from the various chromatograms is shown for the peak concentration tube and its surrounding 10 tubes. Statistical analysis shows that all 11 mean values are not statistically different from each other and thus, at least in terms of FFA/bile acid composition, the mixed micelles appear to be homogeneous.

Whereas gel permeation chromatography gave data as to the homogeneity of size and composition of post-

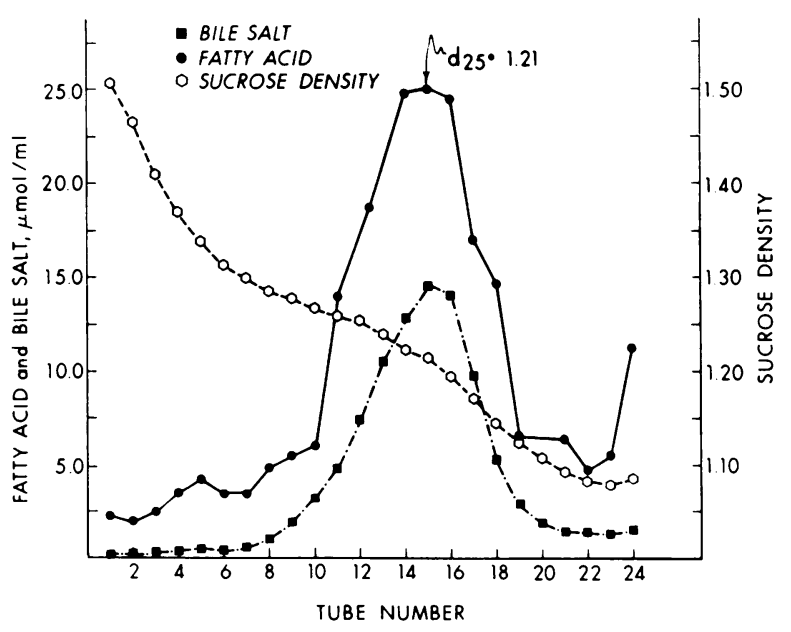

FigURE 8 Linear sucrose density gradient of $1.5-\mathrm{nm}$ retentate in which the sucrose was dissolved in $1.5-\mathrm{nm}$ filtrate. 
prandial mixed micelles, sucrose density centrifugation provided an independent method of assessing the homogeneity of the micelles and was therefore performed on the $1.5-\mathrm{nm}$ retentate of three subjects.

Fig. 8 depicts a representative sucrose density gradient experiment utilizing $1.5-\mathrm{nm}$ retentate from one collection period of one normal. Both bile acid and FFA have distinct concentration peaks which are of the same density. The density for this population of micelles is 1.21 , and that for all collection periods assayed averaged $1.25 \pm 0.03$. Equally as important as providing data about density is that, in each density gradient experiment, the bile acid and FFA concentration peaks coincided. This provides evidence that the bile acid and lipid constituents not only exist in the same-sized particles but also that they are aggregated together in the same particles per se. The increment of FFA in tube no. 24 probably represents some breakdown of micelles and the resultant flotation of non-bile acid-associated FFA. The density of linoleate and taurodeoxycholate was determined by the same techniques as described in Methods for the material obtained in vivo. The density of the mixed micelles of this in vitro experiment was 1.19 .

\section{DISCUSSION}

The mechanism by which dietary lipid has been presumed to be solubilized in the AP of postprandial upper intestinal intraluminal fluid has been the bile acidlipid mixed micelle (29). The only previous evidence for the existence of the micelle has been the demonstration, in ultracentrifuged postprandial intraluminal fluid, of an isotropic phase rich in bile acid and lipid $(2,7,8)$ This "micellar phase," henceforth referred to as "aqueous phase" (AP) is actually a mixture of micelles together with the monomer forms of their constituent bile acids and lipids. This report describes a method for the separation of aggregated from monomer forms in the AP enabling an evaluation of the size, density, and stoichiometry of association of the intestinal mixed micelle.

Previous in vivo studies in humans on the role of bile acid in the solubilization of dietary fat have employed centrifugation $(2,7,8)$ or ultrafiltration (9) to isolate the AP. Each method, including our own, is associated with artifacts. In 1964, Hofmann and Borgström (2) were the first to isolate and describe the gross characteristics of the AP. Their method employed immediate heating of the intestinal fluid to $70^{\circ} \mathrm{C}$ to inactivate lipase. This approach, used subsequently by several other investigators, has been demonstrated to generate FFA during the time necessary to reach $70^{\circ} \mathrm{C}$ (9). In addition, prolonged centrifugation has been shown to displace much of the AP bile acid and FFA from the top of the centrifuge tube toward the bottom
(9). The effects of this perturbation on the aqueous is unknown.

Porter and Saunders in 1971 (9) described an ultrafiltration approach which rapidly yielded an essentially triglyceride-free 100 -nm filtrate from whole postprandial duodenal fluid. Because centrifugation was not employed, clogging of the filters was a problem that required utilization of an essentially protein-free liquid test meal. Furthermore, because $100 \mathrm{~nm}$ was the most discriminating filter used, microemulsions, if present, would be included in the filtrate and, with the techniques employed, could not be identified as being present in addition to micelles.

To avoid some of the limitations and potential artifacts of the above methods, while allowing the study of the physicochemical characteristics of in vivo bile acid-lipid mixed micelles, a method combining ultracentrifugation, ultrafiltration, and gel permeation chromatography has been employed. Potential sources of error with this method are $(a)$ spontaneous lipolysis and phospholipolysis, (b) CMC inaccuracy, (c) adherence of constituents to the filters and gel, and $(d)$ development of a concentration gradient within the AP upon centrifugation.

Lipolysis is unavoidable with the present technique and is clearly represented by an artifactual increment in fatty acid both in whole duodenal fluid and A.P. This increase would not be expected to have a gross effect on the results reported because DDF would be saturated with fatty acid as regards its partition into the aqueous even if reduced by the $35 \%$ shown in Table II. Further, in the aqueous, where monoglyceride would be the expected predominant glyceride available for lipolysis, its product of hydrolysis, fatty acid, behaves relatively similarly with respect to bile acid-lipid mixed micelle formation (29). 2-Monoglyceride, the normal isomer found in postprandial duodenal fluid, is not usually considered a substrate of pancreatic lipase (30). However, the prolonged exposure in these studies of the AP to room temperature would be expected to have resulted in significant isomerization to 1-monoglyceride (31), a suitable substrate for lipase. Although our observations are limited on this point, the monoglyceride concentration was in fact found to be reduced in the unfrozen as compared to quick-frozen samples. The effect of phospholipolysis, although potentially more important because of the different interaction of lecithin vs. lysolecithin with water (27), is somewhat minimized by the findings that $55 \%$ of these phospholipids were lysolecithin in the immediately frozen DDF and because their concentration is low compared to fatty acid.

The CMC of a bile acid can be affected by high concentrations of bile acid (26) and/or its associated 
counterions (27). This is a second potential source of error during the micelle concentration step. In theory, changes in the CMC secondary to changes in amphiphile concentration are small (32), but the CMC of bile acid in in vitro mixtures has been reported to increase with increasing bile acid concentration (26). This phenomenon was observed to ocur in the present study, but the average ultrafiltrate concentration was quite close to theoretical values. These average values might reflect the opposite effect of the associated increased counterion concentration, whose effect would be to lower the CMC of bile acids (27).

The third potential source of error is the amount of adsorption of the bile acid and lipid to the filters and gel columns. This was evaluated in the present study by calculating the recovery of the various constituents as they passed through the two filters and the Sepharose column. The recovery data shows that only a modest amount of bile acid, lipid, and protein is unaccounted for in the filtration and chromatography steps.

It has been suggested that gel columns are inappropriate for the study of bile acid-lipid mixed micelles because of bile acid trapping by the gel (33). In previous studies, (34-37), with the exception of those from Borgström's laboratory $(11,38)$, bile samples were applied to gel columns which were preequilibrated and eluted with buffers which did not contain bile acids. Under these conditions it would be expected that bile acids would diffuse into the eluent at their monomer concentrations leading to a reduction in the concentration of aggregated bile acid and hence the development of a solution supersaturated with lipid. Indeed, experiments performed in this manner have shown the appearance of an opalescent eluent, rich in lipid, at the void volume, disassociated from bile acids which eluted later in the chromatograms. That this disassociation can be avoided by both preequilibration and elution of such columns with bile acid in the buffer was first shown by Bouchier and Cooperband (37). A different problem was observed by Norman (39). Using Sephadex, he found actual adsorption of bile acid to the gel which was manifested by the appearance of bile acid beyond the salt volume of the column. In the present experiments using agarose gel, the columns were preequilibrated and eluted with bile acid and lipid constituents at concentrations slightly above their CMC (1.5-nm filtrate). Under these conditions opalescence was not observed at the void volume of the column and fatty acid/bile acid ratios remained constant. Furthermore, bile acid and lipids assayed penetrated the gel, were eluted before the salt volume, and were recovered quantitativly from the column.

Concentration gradients which developed on centrifugation are a fourth potential source of error (9).
They were small and even further minimized by utilizing the entire AP (see Methods) for the subsequent separation steps.

Although there are limitations in the methodology employed, the present studies give information about the size, density, and stoichiometry of association of the postprandial mixed micelle.

The mixed micelles' size range of $23-35 \mathrm{~nm}$ rules out the possibility of these aggregates being either liquid crystals or a microemulsion and suggests that they are somewhat larger than pure dihydroxy bile acid micelles $(1.6-2.4 \mathrm{~nm})(11,12)$ and considerably larger than trihydroxy bile acid micelles. That the bile acid-lipid mixed micelles are larger than pure bile acid micelles is presumably due to the well-documented effect of incorporation of lipid significantly enlarging the dimensions of the micelle $(38,40)$. It is of interest that our values for Stokes radii are similar to those described for bile acid-lipid mixed micelles in an in vitro system $(38,40)$. In the in vivo situation reported here, it is apparent that the aqueous is saturated with FFA, and therefore, the stated size of the micelles is likely to be maximal for this system.

The density of the postprandial mixed micelle of 1.25 is similar to the observed density of the in vitro deoxycholate micelle (1.15-1.31) (41) and of the linoleatetaurodeoxycholate micelle (1.19). The lipid present in the mixed micelle whose density is $<1$ apparently has little effect on this type of densitometric measurement. This might be due to the small apparent specific volume of bile acid micelles (41) or to other factors (42).

In the postprandial mixed micelle, which is saturated with fatty acid, the stoichiometric relationship of FFA with respect to total bile salt is 1.4 to 1 . This is similar to the saturation ratio of the monolein/bile acid system as studied by Hofmann (1.4-1.7/1, depending on the bile acid) (43).

The postprandial micelle contains less phospholipid (lysolecithin) and cholesterol per mole bile acid on the basis of the stoichiometric data than does fresh human gall bladder bile (44). This reduced proportion of phospholipid and cholesterol is presumably due to the presence in the meal, and hence in DDF, of significant amounts of other lipids, which compete for entry into the mixed micelle. The regression lines related to this data demonstrate as well the dependence of solubilized lipid upon bile acid concentration in the mixed micelle. The composition of the postprandial mixed micelle is also influenced by the oil/AP partitioning of the various lipid species and the relative rates at which they are absorbed. The role of both total glyceride, in general, and monoglyceride in particular, in the bile acidlipid mixed micelle is not documented here, but, at 
least on a molar percent basis, has been found by us and others to be less important than FFA (2).

These studies present the application of several physicochemical methods to the study of the interaction of dietary (and endogenous) lipid components with the predominantly AP present in the intestinal lumen. Their ultimate value as regards the understanding of this complex system will be in delineating more clearly abnormalities manifested by patients who have alterations of one or more of the system's components and in identifying compensatory mechanisms that might in some way limit the steatorrhea that might otherwise be expected in such individuals.

\section{ACKNOWLEDGMENTS}

Dr. Phillip McHale assisted us in performing the statistical analysis. Mary Cox and Robert Pearlstein gave expert technical assistance.

This work was supported in part by Veterans Administration grant no. 5438-01 and National Institutes of Health grant 5T01AM05093-16.

\section{REFERENCES}

1. Borgström, B., A. Dahlqvist, G. Lundh, and J. Sjövall. 1957. Studies of intestinal digestion and absorption in the human. J. Clin. Invest. 36: 1521-1536.

2. Hofmann, A. F., and B. Borgström. 1964. The intraluminal phase of fat digestion in man: the lipid content of the micellar and oil phases of intestinal content obtained during fat digestion and absorption. J. Clin. Invest. $43: 247-257$.

3. Hoffman, N. E., and A. F. Hofmann. 1973. A comparison of the rate of absorption of micellar and nonmicellar oleic acid. Am. J. Dig. Dis. 18: 489-492.

4. Hoffman, N. E., W. J. Simmonds, and R. G. H. Morgan. 1971. A comparison of absorption of free fatty acid and $\alpha$-glyceryl ether in the presence and absence of a micellar phase. Biochim. Biophys. Acta. 231: 487495.

5. Hoffman, N. E. 1970. The relationship between uptake in vitro of oleic acid and micellar solubilization. Biochim. Biophys. Acta. 196: 193-203.

6. Lee, K. Y., D. M. Hurley, and W. J. Simmonds. 1974. The dependence of lipid absorption in vivo on solubilized concentration. Biochem. Biophys. Acta. 337: 214-224.

7. Van-Deest, B. W., J. S. Fordtran, S. G. Morawski, and J. D. Wilson. 1968. Bile salt and micellar fat concentration in proximal small bowel contents of ileectomy patients. J. Clin. Invest. 47: 1314-1324.

8. Krone, C. L., E. Theodor, M. H. Sleisenger, and G. Jeffries. 1968. Studies on the pathogenesis of malabsorption. Medicine (Baltimore). 47: 89-106.

9. Porter, H. P., and D. R. Saunders. 1971. Isolation of the aqueous phase of human intestinal contents during the digestion of a fatty meal. Gastroenterology. 60: 997-1007.

10. Vogel, W. C., and L. Zieve. 1960. A lecithinase A in duodenal contents of man. J. Clin. Invest. 39: 1295-1301.

11. Borgstrom, B. 1965. The dimensions of the bile salt micelle. Measurements by gel filtration. Biochem. Biophys. Acta. 106: 171-183.

12. Kratohvil, J. P., and H. T. DelliColli. 1970. Measurement of the size of micelles: the case of solium taurodeoxycholate. Fed. Proc. 29: 1335-1342.
13. Stempfel, R. S., Jr., and J. B. Sidbury Jr. 1964. Studies with the hydroxysteroid dehydrogenases. I. A simplified method for the enzymatic estimation of 3- and 17-hydroxysteroid. J. Clin. Endocrinol. Metab. 24: 367-374.

14. Folch, J., M. Lees, and G. H. Sloane Stanley. 1957. A simple method for the isolation and purification of total lipids from animal tissues. J. Biol. Chem. 226: 497-509.

15. Mozinger, F. 1965. Photometric determination of Dole's microdetermination of free fatty acids. J. Lipid Res. 6: 157-159.

16. Amenta, J. S. 1963. A rapid chemical method for quantification of lipids separated by thin-layer chromatography. J. Lipid Res. 5: 270-272.

17. Chen, P. S., Jr., H. Warner, and T. Y. Toribara. 1956. Microdetermination of phosphate. Anal. Chem. 28: 17561758.

18. Ames, B. N., and D. T. Dubin. 1960. The role of polyamines in the neutralization of bacteriophage deoxyribonuclic acid. J. Biol. Chem. 235: 769-775.

19. Mansbach, C. M., II. 1973. Complex lipid synthesis in hamster intestine. Biochim. Biophys. Acta. 296: 386-402.

20. Abell, L. L., B. B. Levy, B. B. Brodie, and F. E. Kendall. 1952. A simplified method for the estimation of total cholesterol in serum and demonstration of its specificity. J. Biol. Chem. 195: 357-366.

21. Lowry, O. H., N. J. Rosebrough, A. Farr, and R. J. Randall. 1951. Protein measurement with the Folin phenol reagent. J. Biol. Chem. 193: 265-275.

22. Kessler, G., and M. Wolfman. 1964. An automated procedure for the simultaneous determination of calcium and phosphorus. Clin. Chem. 10: 686-703.

23. Ackers, G. K. 1967. A new calibration procedure for gel filtration columns. J. Biol. Chem. 242: 3237-3238.

24. Tanford, C., Y. Nozaki, J. A. Reynolds. and S. Makino. 1974. Molecular characterization of proteins in detergent solutions. Biochemistry. 13: 2369-2376.

25. Scheffé, N. 1959. The Analysis of Variance. John Wiley \& Sons, Inc., New York.

26. Shankland, W. 1970. The equilibrium and structure of lecithin-cholate mixed micelles. Chem. Phys. Lipids. 4: 109-130.

27. Carey, M. C., and D. M. Small. 1972. Micelle formation by bile salts. Arch. Intern. Med. 130: 506-527.

28. Hofmann, A. F., and D. M. Small. 1967. Detergent properties of bile salts: correlation with physiological function. Annu. Rev. of Med. 18:333-376.

29. Hofmann, A. F., and B. Borgström. 1962. Physicochemical state of lipid in intestinal content during their digestion and absorption. Fed. Proc. 21: 43-50.

30. Entressangles, B., H. Sari, and P. Desnuelle. 1966. On the positional specificity of pancreatic lipase. Biochim. Biophys. Acta. 125: 597-600.

31. Hofmann, A. F. 1963. The behavior and solubility of monoglycerides in dilute micellar bile-salt solution. Biochim. Biophys. Acta. 70: 306-316.

32. Tanford, C. 1973. The Hydrophobic Effect. John Wiley \& Sons, Inc., New York.

33. Carey, M. C., and D. M. Small. 1970. The characteristics of mixed micellar solutions with particular reference to bile. Am. J. Med. 49: 590-608.

34. Thureborn, E. 1963. A water-soluble lipid complex obtained in the macromolecular phase by gel-filtration of human bile. Nature (Lond.). 197: 1301-1302.

35. Neiderhiser, D. H., H. P. Roth, and L. T. Webster, Jr. 1964. Isolation and characterization of a cholesterollecithin complex from bile. J. Clin. Invest. 43: 1252. (Abstr.) 
36. Nakayama, F., and H. Miyake. 1965. Cholesterol complexing by micromolecular functions of human gall bladder bile. J. Lab. (lin. Med. 65: 638-648.

37. Bouchier, I. A. D., and S. R. Cooperband. 1967. Sephadex filtration of a micromolecular aggregate associated with bilirubin. Clin. Chim. Acta. 15: 303-313.

38. Feldman, E. B., and B. Borgstrom. 1966. Phase distribution of sterols: studies by gel filtration. Biochim. Biophys. Acta. 125: 136-147.

39. Norman, A. 1964. Application of gel filtration of bile acids for studies of lipid-complexes in bile. Proc. Soc. Exp. Biol. Med. 116: 902-905.

40. Feldman, E. B., and B. Borgström. 1966. The behavior of glyccride-fatty acid mixtures in bile salt solution: studies by gel filtration. Lipids. 1: 430-438.

41. Small, D. M. The physical chemistry of cholanic acils. 1971. In The Bile Salts. P. P. Nair and D. Kritchevsky, editors. Plenum Publishing Corporation, New York. 249-354.

42. Lafont, H., and J. Hauton. 1973. Is a peptidic fraction naturally associated with bile lipids? Helv. Med. Acta. 37 : 137-141.

43. Hofmann, A. F. 1963. The function of bile salts in fat absorption. Biochem. J. 89 : 57-68.

44. Admirand, W. H., and D. M. Small. 1968. The physicochemical basis of cholesterol gallstones formation in man. J. Clin. Inz'cst. 7: 1043-1052. 